\title{
Tecnoestrés en población mexicana y su relación con variables sociodemográficas y laborales
}

\section{Technostress in the Mexican population and its relationship with sociodemographic and labor variables}

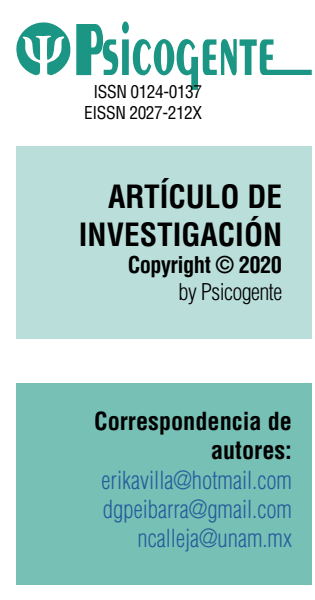

Recibido: $13-06-2019$ Aceptado: 04-04-2020 Publicado: 05-06-2020
Erika Villavicencio-Ayub (iD - Diana Guadalupe Ibarra Aguilar (iD) - Nazira Calleja (iD Universidad Nacional Autónoma de México Ciudad Universitaria, México

\section{Resumen}

Objetivo: Medir la relación entre variables sociodemográficas, variables laborales y la frecuencia del uso de las tecnologías, con el nivel de tecnoansiedad, tecnofatiga y tecnoadicción como experiencias de tecnoestrés, en una muestra de adolescentes, jóvenes y adultos mexicanos.

Método: Se aplicaron las escalas para medir tecnoestrés y tecnoadicción de Cazares \& Villavicencio (2019) y un cuestionario de variables sociodemográficas y laborales de elaboración propia, a un total de 981 participantes mexicanos entre 13 y 69 años, utilizando la herramienta de formularios de Google. Los datos obtenidos fueron sometidos a un análisis de nivel descriptivo, correlacional e inferencial (Kerlinger \& Lee, 2002), con ayuda del programa estadístico SPSS v.20.

Resultados: Respecto a la tecnofatiga, los resultados arrojaron diferencias significativas por sexo $(U=$ $104026.50, p=.037)$ y antigüedad en el trabajo $\left[x^{2(3)}=11.213, p=.011\right]$ y una significancia marginal por estado civil $(U=78329.00, p=.058)$. En cuanto a la tecnoadicción, se encontraron diferencias significativas por estado civil $(U=76121.50, p=.012)$ y ocupación $\left[\chi^{2(2)}=4.698, p=.008\right]$. Con relación a la tecnoansiedad, fueron arrojadas diferencias significativas por tipo de empresa $(U=61348.00, p=.049)$ Finalmente, se encontró que las personas con mayor nivel de escolaridad, los directivos y los empresarios independientes, presentaron mayor tecnoansiedad, tecnofatiga y tecnoadicción.

Conclusiones: Los resultados permiten afirmar que en México existe la presencia de tecnoestrés. Además, se demuestra la relación entre las experiencias del tecnoestrés y variables sociodemográficas (sexo, estado civil, ocupación y escolaridad) y laborales (antigüedad laboral, tipo de empresa y nivel de puesto). Los resultados constituyen las primeras aportaciones de la investigación del tecnoestrés en México, país que impulsa el uso de la tecnología.

Palabras clave: tecnoestrés, tecnoansiedad, tecnofatiga, tecnoadicción, TIC, tecnología.

Abstract

Objective: To measure the relationship between sociodemographic variables, labor variables, and technology frequency of use with the level of technostress experiences such as techno-anxiety, techno-fatigue, and techno-addiction, using a sample of Mexican adolescents, youth, and adults.

Method: The participants of the study were 981 Mexican nationals aged 13-69. The scales implemented to measure technostress and techno-addiction were the ones used in Cazares \& Villavicencio (2019) and a sociodemographic and labor variables questionnaire created by the authors, implemented using Google's form tool. Data obtained were subjected to a descriptive, correlational, and inferential level analysis (Kerlinger \& Lee, 2002), using the statistical software SPSS v.20.

Results: Regarding techno-fatigue, the results showed significant differences on the basis of sex (U $=104026.50, p=0.037)$ and seniority $\left[\chi^{2(3)}=11.213, p=0.011\right]$ and a marginal significance on the basis of marital status $(U=78329.00, p=.058)$. Regarding techno-addiction, significant differences were found on the basis of marital status $(U=76121.50, p=0.012)$ and occupation $\left[X^{2(2)}=4.698, p=\right.$ 0.008]. Regarding techno-anxiety, significant differences were found on the basis of company type ( $U=$ $61348.00, p=0.049$ ). Finally, it was found that people who received better schooling, such as directors 
and independent entrepreneurs, showed greater techno-anxiety, techno-fatigue, and techno-addiction. Conclusions: The results confirm that technostress is prevalent in Mexico. In addition, there is a confirmed relationship between technostress experiences, sociodemographic variables (sex, marital status, occupation, and schooling), and labor variables (seniority, company type, and position level). The results constitute the first technostress research contributions in Mexico, a country that encourages the use of technology.

Keywords: technostress, techno-anxiety, techno-fatigue, techno-addiction, ICT, technology

\section{INTRODUCCIÓN}

El avance tecnológico está impactando en un nivel social, económico y psicológico a las organizaciones (West \& Saker, 2012; Sigala, 2018; Yu, Ndumu, Mon \& Fan, 2018); modificando la dinámica de los centros de trabajo (Salanova, Cifre \& Martin, 2004; Mueller, 2006; Salanova, Llorens, Cifre \& Nogareda, 2007; Alfaro de Prado, 2008; Yan, Guo, Lee \& Vogel, 2013; Khuntia, Tanniru \& Weiner, 2015; De Wet, Koekemoer \& Nel, 2016; Haddara \& Hetlevik, 2016; Duke \& Montag, 2017; Stich, Tarafdar, Stacey \& Cooper, 2018; Yin, Ou, Davison $\& W u, 2018$ ) y de los espacios educativos (Allan \& Lawless, 2003; Abrahams, 2010; Brooks, 2015; Hsiao, Shu \& Huang, 2017; Al-Yafi, El-Masri \& Tsai, 2018; Khatri, Samuel \& Dennis, 2018; Cao, Masood, Luqman \& Ali, 2018).

Entre las ventajas de las innovaciones tecnológicas se encuentran la oportunidad de realizar múltiples actividades, independientemente del tiempo y el espacio (Çoklar \& Sahin, 2011); nuevas formas de trabajo, como el e-work (Grant, Wallace, Spurgeon, Tramontano \& Charalampous, 2019); la facilidad de acceso a documentos y sistemas, mejorando el intercambio de ideas (De Wet et al., 2016; Guillén, 2016; Cao \& Yu, 2019); y el aumento de la calidad y la productividad (Alfaro de Prado, 2008). Sin embargo, se debe denotar que "las TIC por sí solas no son garantía de éxito" (Alfaro de Prado, 2008, p. 18) debido a que son las personas quienes las utilizan (Brooks \& Califf, 2017; Gaudioso, Turel \& Galimberti, 2017).

A la par, estudios han encontrado que las TIC incrementan la demanda de trabajo y producen una dificultad para desconectarse de las tareas laborales, aun estando en casa, creando la expectativa de siempre estar disponibles (Alfaro de Prado, 2008; De Wet et al., 2016; Seong \& Park, 2016; Carlotto, Welter \& Jones, 2017; Yao \& Cao, 2017). Además, la constante y rápida renovación de herramientas tecnológicas es percibida como una fuente de estrés (Sami \& Pangannaiah, 2006; Çoklar \& Sahin, 2011; Haddara \& Hetlevik, 2016) y de distracción (Brooks, 2015; De Wet et al., 2016; Hsiao et al., 2017; Hsiao, 2017). 
Ligado a estos resultados, Brod (1984, cit. en Salanova et al., 2007) es el primero en afirmar que existe "una enfermedad de adaptación causada por la falta de habilidad para tratar con las nuevas tecnologías del ordenador de manera saludable" (p.1), a la que denomina tecnoestrés. Se habla de tecnoestrés para referirse a los efectos negativos psicológicos, sociales y biológicos, del uso de las tecnologías (Agogo \& Hess, 2015; Coppari et al., 2018). Asimismo, se ha reportado que existen tres experiencias distintas del tecnoestrés: la tecnoansiedad, la tecnofatiga y la tecnoadicción (Salanova et al., 2007) (Véase Tabla 1).

Tabla 1

Experiencias de tecnoestrés

\section{TECNOANSIEDAD}

La persona experimenta altos niveles de activación fisiológica no placentera, y siente tensión y malestar por el uso presente o futuro de algún tipo de TIC. La misma ansiedad lleva a tener actitudes escépticas respecto al uso de tecnologías, a la vez que pensamientos negativos sobre la propia capacidad y competencia con las TIC.

Fuente: Tabla creada con información recuperada de Salanova et al., 2007.

Se caracteriza por sentimientos de cansancio y agotamiento mental y cognitivo debido al uso de tecnologías, complementados también con actitudes escépticas y creencias de ineficacia con el uso de TIC.
Se define por la incontrolable compulsión a utilizar las TIC en "todo momento y lugar", y durante largos períodos de tiempo.

Posteriormente se habla de solo dos tipos de tecnoestrés (tecnostrain y tecnoadicción) debido a que se considera que la ansiedad y la fatiga son componentes de la experiencia tecnostrain (Guillén, 2016). Tanto el rechazo como el uso compulsivo de las TIC denotan un comportamiento disfuncional (Alfaro de Prado, 2008; Zhang, Zhao, Lu \& Yang, 2016; Hsiao et al., 2017; Luqman, Cao, Ali, Masood \& Yu, 2017; Cao et al., 2018; Dhir, Yossatorn, Kaur \& Chen, 2018; Stich et al., 2018; Tams, Legoux \& Leger, 2018).

En años recientes, se ha incrementado el número de investigaciones sobre tecnoestrés debido a las consecuencias que este conlleva (Quinn, 2001; Brooks \& Califf, 2017; Gaudioso et al., 2017; Hsiao et al., 2017; Krishnan, 2017; Martínez-Corcoles, Teichmann \& Murdvee, 2017; Steelman \& Soror, 2017; Cao \& Sun, 2018; Loderer, Pekrun \& Lester, 2018; Loiacono \& McCoy, 2018; Yu, Cao, Liu \& Wang, 2018).

Una persona con tecnoestrés, fisiológicamente hablando, padece ansiedad, irritabilidad, dolor de cabeza (Alfaro de Prado, 2008; Khasawneh, 2018a; Khasawneh, 2018b); síndrome del túnel carpiano, dolores musculares (Llorens, Salanova \& Ventura, 2011); problemas de sueño (Thomee, Eklof, Gustafsson, Nilsson \& Hagber, 2007; Çoklar \& Sahin, 2011); aumento en 
la producción de las hormonas relacionadas con la aparición de estrés, adrenalina y cortisol (McEwen, 2006; Riedl, 2013).

A nivel psicosocial, se ha identificado que cuando los usuarios de las TIC valoran la exposición a la tecnología como negativa pueden presentar burnout (Salanova \& Schaufeli, 2000), y cuando su valoración es positiva muestran mayor engagement (Salanova \& Llorens, 2009). A su vez, el uso excesivo de la tecnología, como en cualquier otra adicción, crea dependencia (Quinn, 2001; Tams et al., 2018; Cao \& Yu, 2019). La sobre-estimulación a la que se puede estar expuesto produce una sobrecarga mental, dificultad para recordar, pensar con claridad y descansar, y menor percepción de felicidad (Brooks, 2015; Picón, Toledo \& Navarro, 2017; Hughes \& Burke, 2018).

A nivel individual, los trabajadores suelen aislarse socialmente, se vuelven irascibles, con cambios de humor, y descuidan no solo la vida laboral sino también la familiar (Llorens et al., 2011; Duke \& Montag, 2017; Jung, Pawlowski \& Kim, 2017), dejando así que el tiempo y la energía que dedican a otras actividades sea menor (Yao \& Cao, 2017; Cao \& Yu, 2019).

Finalmente, si en una organización se experimenta el tecnoestrés, hay un decremento del desempeño de los trabajadores (Brooks, 2015; Yu et al., 2018; Cao \& Yu, 2019), de las horas trabajadas (Duke \& Montag, 2017), y de la satisfacción laboral (Ragu-Nathan, Tarafdar, Ragu-Nathan \& Tu, 2008; Haddara \& Hetlevik, 2016; Seong \& Park, 2016; Yin et al., 2018). También, se experimenta un aumento en el índice de ausentismo, rotación, bajas laborales, reducción de productividad y un menor compromiso laboral (Brooks \& Califf, 2017; Stich et al., 2018).

Los investigadores se han propuesto identificar las características de los usuarios de la tecnología y cómo estas impactan en el nivel de tecnoestrés, una de las variables mejor estudiadas es la edad. Gran parte de los estudios afirman que sí existen diferencias en cuanto la experiencia de tecnoestrés y la edad, por ejemplo, se menciona que las personas mayores padecen fatiga, ansiedad, tienen más creencias de ineficacia, un mayor escepticismo (Salanova et al., 2007; Hill, Davies \& Williams, 2008; Çoklar \& Sahin, 2011; Zhang et al., 2016; Picón et al., 2017), y rechazan la idea de utilizar las TIC (Mattila, Karjaluoto \& Pento, 2003; Alfaro de Prado, 2008). Sin embargo, lo anterior se pone en duda cuando otra investigación afirma que, los adultos 
mayores utilizan la experiencia que tienen enfrentando otras situaciones de estrés dentro de su lugar de trabajo, para disminuir sus niveles de tecnoestrés (Ragu-Nathan et al., 2008). Asimismo, se tiene registro de que los jóvenes adultos y adultos son la población que tiende a sufrir tecnoestrés, mayor fatiga, ansiedad e ineficacia (Carlotto et al., 2017; Picón et al., 2017).

Otro de los resultados relacionado a la edad, asegura que los jóvenes tienden a usar con mayor frecuencia las TIC, una de las razones que se ha encontrado para explicar esto es que les permiten permanecer en el anonimato (Gaspar, 2016; Hsiao, 2017). Además, reportan ser los que más mensajes envían (Teo, 2001), siendo Whatsapp una de las principales aplicaciones para ellos (Montag et al., 2015). A pesar de esto, otra investigación afirma que, a causa de que la tecnología ya es de uso cotidiano y frecuente, no hay diferencias significativas en cuanto a la edad, en el grado de aceptación de la misma (Khasawneh, 2018b).

La experiencia de tecnoestrés entre hombres y mujeres también se ha estudiado, por ejemplo, algunas investigaciones argumentan que son las mujeres las que manifiestan mayores niveles de ansiedad y fatiga con el uso de las TIC, mostrando actitudes más negativas y sentimientos de poca capacidad para emplearlas (Salanova et al., 2007; Baloglu \& Cevik, 2008; Carlotto et al., 2017; Picón et al., 2017). También se ha afirmado que las mujeres suelen utilizar la tecnología con el fin de socializar o comunicar, siendo las que más mandan mensajes (Teo, 2001; Lee, Chang, Lin \& Cheng, 2014), las que más utilizan la aplicación Whatsapp (Montag et al., 2015), y las que más apegadas están del celular (Lee et al., 2014). Otros investigadores afirman que son los hombres quienes experimentan más tecnoestrés (Ragu-Nathan et al., 2008) y fatiga (Zhang et al., 2016; Carlotto et al., 2017) que las mujeres. Esto debido a que los hombres muestran una mayor disposición a usar las TIC incluso cuando no las necesitan (Carlotto et al., 2017) o bien cuando se trata de su desarrollo personal (Shao, 2018); además, suelen jugar en el celular con mayor frecuencia (Hsiao, 2017) y realizan descargas y compras online (Teo, 2001). Los hombres reportan ser más compulsivos con el uso de las tecnologías (Lee et al., 2014).

En ese mismo orden de ideas, otros estudios muestran que no existen diferencias significativas en la cantidad de compras que realizan hombres y mujeres (Dhanapal, Vashu \& Subramaniam, 2015), en cuanto al grado de 
aceptación de la tecnología (Khasawneh, 2018a), ni en el nivel de adicción al celular (Duke \& Montag, 2017; Hsiao et al., 2017). Un aspecto a considerar en este tipo de investigaciones es la cultura, ya que esta puede propiciar o limitar el acceso a la tecnología y por lo tanto modular la experiencia tanto de hombres como de mujeres con respecto a las TIC (Ahmad, Rafiq \& Ahmad, 2018).

La investigación del uso de la tecnología no ha profundizado en las diferencias generacionales. Gracias a los pocos estudios que se tienen al respecto se sabe que tanto generación BabyBoomer, X y Y realizan compras por internet, sin embargo la generación BabyBoomer lo hace en menor medida (Dhanapal et al., 2015). Los jóvenes, también llamados como generación APP, nativos digitales, millennials y centennials, son los que pasan más tiempo utilizando la tecnología. La generación $\mathrm{X}$, conformada por el adulto intermedio, es la que más padece de tecnoestrés ya que se debe enfrentar a un desarrollo acelerado de la tecnología, evitando ser rezagado por la generación $\mathrm{Y}$ que está mejor adaptada a los cambios que esta plantea (Coppari et al., 2017).

En cuanto al estado civil se encontró un estudio que afirma que los trabajadores casados presentan mayores niveles de escepticismo y fatiga, y los solteros padecen niveles altos de ansiedad e ineficacia (Carlotto et al., 2017).

La educación está asociada negativamente con el uso diario del celular (Montag et al., 2015). El tecnoestrés en general disminuye conforme el nivel educativo aumenta (Ragu-Nathan et al., 2008). Por otra parte, los estudiantes jóvenes tienden a utilizar con gran frecuencia la tecnología, creando la posibilidad de que presenten adicción a la misma. Un estudio ha reportado que cuando el uso de la tecnología es normal, el desempeño escolar tiende a ser mayor en comparación con aquellos que hacen un bajo o un alto uso de esta (Al-Yafi et al., 2018).

En general, las personas que se dedican a estudiar y que son menores de 20 años tienden a padecer menos tecnoestrés que otras personas que, por ejemplo, se dedican a la granja, que ya están jubilados, o que se encargan de las labores del hogar (Sonya, 2003).

Si hablamos de variables laborales como el nivel de puesto, el tipo de empresa o la antigüedad con relación al uso de la tecnología, la información 
que se tiene es escasa. Oh \& Sungbum (2016) encontraron que entre más alta sea la posición que se ocupa menor percepción de carga de trabajo se tiene e incluso no se le da importancia al hecho de trabajar en vacaciones. Mientras que, para las personas con un puesto más operativo la cantidad de horas laboradas es muy importante. También, se reconoce que la antigüedad puede jugar a favor gracias a la experiencia que se adquiere para manejar situaciones estresantes (Ragu-Nathan et al., 2008).

Cuando una persona utiliza la tecnología con mayor frecuencia tiende a poseer mayor experiencia en su manejo, lo que puede disminuir sus niveles de tecnoansiedad (Çoklar \& Sahin, 2011; Picón et al., 2017). Sin embargo, el uso excesivo de la tecnología lleva a altos niveles de tecnoestrés (Brooks, 2015; Carlotto et al., 2017).

En México, la incorporación de las tecnologías a la vida cotidiana de sus habitantes se ha impulsado por distintos programas cuyo objetivo es erradicar la brecha digital, por ejemplo, México conectado (Gobierno de la CDMX, 2019), Programa de Inclusión y Alfabetización Digital PIAD (México Digital, 2014), Internet gratis en el metro (Gobierno de la CDMX, 2018). Sin embargo, la revisión de la literatura sobre tecnoestrés no arroja estudios científicos realizados con población mexicana, los cuales sometan a análisis la presencia del mismo y las características de las personas que lo experimentan. Es por esto, que el objetivo de esta investigación es identificar la relación de variables sociodemográficas (edad, generación, sexo, estado civil, escolaridad), variables laborales (tipo de puesto y de empresa, antigüedad en el trabajo) y la frecuencia del uso de las TIC, con el nivel de tecnoestrés; aportando un primer estudio en México sobre este fenómeno que permita comenzar a caracterizar a las personas que interactúan con la tecnología y que además sirva de base para futuras investigaciones.

\section{MÉTODO}

\subsection{Diseño}

El estudio tuvo un diseño no experimental y de tipo transversal exploratorio, esto implica que no se realizó ninguna manipulación de las variables ni ningún tipo de intervención con los participantes (Kerlinger \& Lee, 2002); además, la recolección de los datos se hizo en un solo momento (Hernández, Fernández \& Baptista, 2014). 


\subsection{Participantes}

Se ocupó un muestreo no probabilístico por conveniencia, con consentimiento informado y anonimato. La muestra del presente estudio se conformó por 981 participantes con edades comprendidas entre 13 y 69 años reportó usar las tecnologías todos los días. A continuación, se muestra el porcentaje de la muestra por categorías (Véase Tabla 2):

Tabla 2

Muestra

\begin{tabular}{|c|c|c|c|}
\hline TIPO DE VARIABLE & VARIABLE & CATEGORÍA & $\%$ \\
\hline \multirow[t]{23}{*}{ Demográficas } & Sexo & Hombres & $37,6 \%$ \\
\hline & & Mujeres & $62,4 \%$ \\
\hline & & Babyboomers & $7 \%$ \\
\hline & *Generación & Generación X & $25,4 \%$ \\
\hline & 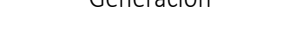 & Generación Millennial & $52,4 \%$ \\
\hline & & Generación Z & $15,2 \%$ \\
\hline & & Soltero & $61,7 \%$ \\
\hline & Estado civil & Casado & $28,6 \%$ \\
\hline & & Otro & $9,7 \%$ \\
\hline & & Primaria & $1,7 \%$ \\
\hline & & Secundaria & $7,5 \%$ \\
\hline & Escolaridad & Técnico-Bachillerato & $14,1 \%$ \\
\hline & Escoldriudu & Licenciatura & $61 \%$ \\
\hline & & Posgrado & $14,6 \%$ \\
\hline & & Otro & $1,1 \%$ \\
\hline & & Trabajador & $88,5 \%$ \\
\hline & Ocupación & Ama de casa & $1,7 \%$ \\
\hline & & Estudiante, becario, pasante, prestador de servicio. & $9,8 \%$ \\
\hline & & Operativo & $53,7 \%$ \\
\hline & & Coordinación & $24,2 \%$ \\
\hline & Puesto desempeñado & Mandos medios & $12,9 \%$ \\
\hline & & Directivos & $3,5 \%$ \\
\hline & & Empresario independiente & $5,8 \%$ \\
\hline \multirow[t]{8}{*}{ Laborales } & Tinn de emnresa & Pública & $23,4 \%$ \\
\hline & 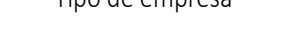 & Privada & $76,6 \%$ \\
\hline & & Menos de un año & $26,6 \%$ \\
\hline & Antig̈̈edad en el nuesto & Entre 1 y 5 años & $43,2 \%$ \\
\hline & Amigueudu en ei puesto & Entre 5 y 10 años & $12,9 \%$ \\
\hline & & Más de 10 años & $17,3 \%$ \\
\hline & & Nunca & $2,2 \%$ \\
\hline & & Un par de veces al mes & $1,1 \%$ \\
\hline \multirow[t]{3}{*}{ Frecuencia de uso } & & Una vez a la semana & $1,0 \%$ \\
\hline & & Un par de veces a la semana & $5,3 \%$ \\
\hline & & Todos los días & $90,3 \%$ \\
\hline
\end{tabular}

* Generación: Babyboomers (nacieron entre 1946 y 1964); Generación X (nacieron entre 1965 y 1981); Generación Millennial (nacieron entre 1982 y 1994); y Generación Z (nacieron entre de 1995 y la actualidad) (Dhanapal et al., 2015). 


\subsection{Técnicas e instrumentos para la recolección de información}

\subsubsection{Cuestionario de variables sociodemográficas y laborales}

Cuestionario de elaboración propia compuesto de 9 reactivos, una pregunta con opción de respuesta abierta (edad) a partir de la cual se calculó la generación según lo postulado por Dhanapal et al. (2015), y ocho más de opción múltiple.

Reactivos de variables sociodemográficas (5): Edad (respuesta libre), sexo (hombre, mujer), escolaridad (primaria, secundaria, técnico-bachillerato, licenciatura, posgrado), estado civil (soltero, casado, otro), ocupación (trabajador, ama de casa, estudiante o becario o pasante o prestador de servicio).

Reactivos de variables laborales (3): puesto desempeñado (operativo, coordinación, mandos medios, directivos, empresario independiente), tipo de empresa (pública, privada), antigüedad en el puesto (menos de un año, entre 1 y 5 años, entre 5 y 10 años, más de 10 años).

Finalmente, se añadió una pregunta para medir la frecuencia de uso de TIC: ¿Con qué frecuencia usa las tecnologías? (nunca, un par de veces al mes, una vez a la semana, un par de veces a la semana, todos los días).

\subsubsection{Escalas para medir tecnoestrés y tecnoadicción en una población} laboral mexicana (Cazares \& Villavicencio, 2019)

Se eligió este instrumento porque es el único adaptado a la población laboral mexicana y cumple con los criterios psicométricos necesarios, es decir, posee una confiabilidad de 0.86 y está validado. Se compone de 20 reactivos con opciones de respuesta tipo Likert en escala de frecuencia (Nunca $=0$, Casi nunca $=1$, Algunas veces $=2$, Regularmente $=3$, Bastante a menudo $=4$, Casi siempre $=5$ y Siempre $=6$ ). Evalúa los niveles de tecnoestrés en dos factores, displacer derivado del uso de TIC y rechazo de TIC; y tecnoadicción con dos factores, uso excesivo y uso compulsivo de la tecnología. Obtuvo una alfa de Cronbach de .89 para la escala de tecnoestrés y .84 para la escala de tecnoadicción en su aplicación. Para el presente estudio, el alfa de Cronbach fue de .86 .

\subsection{Procedimiento}

Se creó un formulario de Google, el cual estuvo conformado por un breve cuestionario de 9 reactivos cuya finalidad fue la recolección de las características sociodemográficas y laborales, así como la frecuencia de uso de las TIC 
de los participantes; además de las escalas de tecnoestrés y tecnoadicción. La distribución del formulario se hizo a través de redes sociales. La participación de las personas fue voluntaria y anónima, con consentimiento informado. Posteriormente, las respuestas fueron registradas y analizadas en el programa estadístico SPSS v.20. Se comenzó por el análisis psicométrico del instrumento para después analizar la relación de las variables sociodemográficas (edad, generación, sexo, estado civil, escolaridad, ocupación), laborales (nivel de puesto desempeñado, antigüedad en el trabajo, tipo de empresa) y la frecuencia del uso de las TIC, con el tecnoestrés.

\subsection{Análisis de datos}

Los datos obtenidos fueron sometidos a un análisis de nivel descriptivo, correlacional e inferencial (Kerlinger \& Lee, 2002). Descriptivo para calcular frecuencias, medias y desviaciones estándar. Se efectuaron análisis correlacionales e inferenciales para evaluar la significancia de la relación de las variables sociodemográficas y laborales, así como de la frecuencia del uso de las TIC, con el nivel de tecnoestrés. Dado que, de acuerdo con la prueba de Kolmogorov-Smirnov, las variables no mostraron una distribución normal $(p<.05)$, se utilizaron las pruebas no paramétricas $U$ de Mann_Whitney (para comparación de dos grupos independientes), H de Kruskal-Wallis (para comparación de más de dos grupos independientes) y correlación de Spearman (Rho).

\section{RESULTADOS}

\subsection{Análisis psicométrico de las Escalas para medir tecnoestrés y} tecnoadicción en una población laboral mexicana (Cazares \& Villavicencio, 2019)

El análisis factorial exploratorio de componentes principales con rotación varimax de las escalas para medir tecnoestrés y tecnoadicción en una población laboral mexicana, arrojó tres factores que explicaron el 46,04 \% de la varianza (véase Tabla 3); el primero se refiere a la tecnoansiedad, entendida como aquella incomodidad, irritabilidad y rechazo del uso presente o futuro de algún tipo de TIC; el segundo habla de la tecnoadicción, definida como el incontrolable impulso a utilizar las TIC en todo momento y lugar; y el tercero señala la tecnofatiga, entendida como sentimientos de cansancio y agotamiento mental y cognitivo debidos al uso de tecnologías. La varianza media extraída (AVE) fue de .436. El coeficiente de confiabilidad alpha de Cronbach para la escala total fue de 0.86 y para los factores resultó superior a 0.80 . 
Los factores correlacionaron significativamente. La correlación más alta se presentó entre los factores tecnoansiedad y tecnofatiga $(r=.562, p<.001)$, y la más baja entre los factores tecnoansiedad y tecnoadicción ( $r=.142, p<.001)$, lo que quiere decir que las personas que padecen tecnoansiedad también tienden a presentar tecnofatiga, pero menos tecnoadicción. Así mismo, se presenta una moderada correlación entre tecnofatiga y tecnoadicción $(r=.431, p<.001)$, lo que implica que las personas con tecnofatiga también tiene probabilidad de sufrir tecnoadicción.

Tabla 3.

Escalas para medir tecnoestrés y tecnoadicción en una población laboral mexicana Factores, cargas factoriales, índices psicométricos y estadísticos descriptivos

\begin{tabular}{|c|c|c|c|c|}
\hline \multirow{2}{*}{\multicolumn{2}{|c|}{ REACTIVOS }} & \multicolumn{3}{|c|}{ FACTORES } \\
\hline & & \multirow{2}{*}{$\begin{array}{c}\text { TECNOANSIEDAD } \\
.540\end{array}$} & \multirow[t]{2}{*}{ TECNOADICCIÓN } & \multirow[t]{2}{*}{ TECNOFATIGA } \\
\hline $\begin{array}{r}\text { 2. Trabajar con tecnologías me hace se } \\
\text { impaciente. }\end{array}$ & table e & & & \\
\hline \multicolumn{2}{|c|}{ 3. Dudo del significado de trabajar con tecnologías. } & .566 & & \\
\hline \multicolumn{2}{|c|}{ 6. Cada vez me siento menos implicado en el uso de las tecnologías. } & .549 & & \\
\hline \multicolumn{2}{|c|}{ 10. Es difícil trabajar con tecnologías. } & .702 & & \\
\hline \multicolumn{2}{|c|}{ 13. Las cosas me salen mal cuando utilizo tecnologías. } & .631 & & \\
\hline \multicolumn{2}{|c|}{ 14. Cada vez más me molesta usar tecnologías en mi trabajo. } & .595 & & \\
\hline \multicolumn{2}{|c|}{ 15. Me siento tenso y ansioso cuando trabajo con tecnologías. } & .588 & & \\
\hline \multicolumn{2}{|c|}{ 18. Prefiero no usar las tecnologías porque entorpecen mi trabajo. } & .506 & & \\
\hline \multicolumn{2}{|c|}{ 19. Me cuesta trabajo aprender a usar nuevas tecnologías. } & .750 & & \\
\hline \multicolumn{2}{|c|}{$\begin{array}{l}\text { 1. Me siento mal si no tengo acceso a las tecnologías (Internet, correo } \\
\text { electrónico, teléfono celular, etc.). }\end{array}$} & & .640 & \\
\hline \multicolumn{2}{|c|}{$\begin{array}{l}\text { 5. Siento que un impulso interno me obliga a utilizar las tecnologías en } \\
\text { cualquier momento y lugar. }\end{array}$} & & .726 & \\
\hline \multicolumn{2}{|c|}{$\begin{array}{l}\text { 8. Me encuentro pensando en tecnologías continuamente (por ejemplo, } \\
\text { en revisar el correo electrónico, buscar información en internet, utilizar } \\
\text { redes sociales etc.) incluso fuera del horario de trabajo. }\end{array}$} & & .716 & \\
\hline \multicolumn{2}{|c|}{$\begin{array}{l}\text { 9. Siento una enorme necesidad de utilizar las tecnologías en momen- } \\
\text { tos que no son adecuados (p. ej., al manejar). }\end{array}$} & & .611 & \\
\hline \multicolumn{2}{|c|}{$\begin{array}{l}\text { 12. Dedico más tiempo a usar las tecnologías que a estar con mis } \\
\text { amigos y familiares. }\end{array}$} & & .573 & \\
\hline \multicolumn{2}{|c|}{$\begin{array}{l}\text { 16. Dedico más tiempo a usar las tecnologías que a practicar algún } \\
\text { deporte o actividad al aire libre. }\end{array}$} & & .559 & \\
\hline \multicolumn{2}{|c|}{ 17. Me gusta pasar largas horas usando tecnologías. } & & .733 & \\
\hline \multicolumn{2}{|c|}{ 20. Me siento incómodo cuando no puedo utilizar tecnologías. } & & .667 & \\
\hline \multicolumn{2}{|c|}{$\begin{array}{l}\text { 4. Me resulta difícil relajarme después de un día de trabajo utilizando } \\
\text { tecnologías. }\end{array}$} & & & -.547 \\
\hline \multicolumn{2}{|c|}{ 7. Es difícil que me concentre después de trabajar con tecnologías. } & & & -.722 \\
\hline \multicolumn{2}{|c|}{$\begin{array}{c}\text { 11. Después de usar tecnologías me cuesta trabajo prestar atención a } \\
\text { otras actividades. }\end{array}$} & & & -.577 \\
\hline \multicolumn{5}{|c|}{ Total } \\
\hline Número de reactivos & 20 & 9 & 8 & 3 \\
\hline \% de la varianza explicada & 46.04 & 27.40 & 15.29 & 3.41 \\
\hline Alfa de Cronbach & .873 & .847 & .858 & .804 \\
\hline
\end{tabular}




\begin{tabular}{cccc}
\hline Correlaciones inter factor & & & \\
Tecnoansiedad & 1 & & \\
Tecnofatiga & $.562^{*}$ & 1 & 1 \\
Tecnoadicción & $.142^{*}$ & $.431^{*}$ & 1 \\
\hline
\end{tabular}

$* p<.001$

Respecto de los puntajes obtenidos por la muestra, la media en todos los factores se ubicó por debajo de la media teórica. La media más alta (2.69 \pm 1.41) correspondió al factor de tecnofatiga y la más baja al de tecnoansiedad (0.95 \pm 0.88$)$ (véase Tabla 4):

Tabla 4

Medias por factores

\begin{tabular}{cccc}
\hline & \multicolumn{3}{c}{ FACTORES } \\
\cline { 2 - 4 } Media (media teórica = 4; rango 0-6) & TECNOANSIEDAD & TECNOADICCIÓN & TECNOFATIGA \\
\hline Desviación estándar & 0.95 & 1.42 & 2.69 \\
\hline
\end{tabular}

\subsection{Relación de variables sociodemográficas con tecnoestrés}

Tras contrastar los puntajes de los factores de tecnoestrés (tecnoansiedad, tecnofatiga y tecnoadicción) por sexo, solo en el factor tecnofatiga las mujeres obtuvieron puntajes significativamente más altos en comparación con los hombres (véase Tabla 5).

Tabla 5

Relación entre el sexo de los participantes y los factores de tecnoestrés

\begin{tabular}{ccc}
\hline CATEGORÍAS & MEDIAS (DS) & $\begin{array}{c}\text { PRUEBA ESTADÍSTICA } \\
\text { U DE MANN-WHITNEY }\end{array}$ \\
\hline TECNOANSIEDAD & & \\
\hline Hombres & $0.89(0.83) ;$ & $U=108207.00, p=.272$ \\
Mujeres & $0.97(0.90)$ & \\
\hline TECNOFATIGA & & $U=104026.50, p=.037$ \\
\hline Hombres & $1.29(1.30)$ & \\
Mujeres & $1.50(1.43)$ & $U=112239.50, p=.875$ \\
\hline TECNOADICCIÓN & & \\
\hline Hombres & $2.71(1.39)$ & \\
Mujeres & $2.70(1.43)$ & \\
\hline
\end{tabular}

La prueba de Kruskal-Wallis mostró que la variable escolaridad afectó significativamente el puntaje de tecnoansiedad $\left[\chi^{2(3)}=18.504, p<.001\right]$, de tecnofatiga $\left[\chi^{2(3)}=38.311, p<.001\right]$ y de tecnoadicción $\left[\chi^{2(3)}=18.348, p<.001\right]$. Se encontró que a mayor escolaridad, mayor tecnoansiedad, tecnofatiga y tecnoadicción. Al hacer comparaciones por pares de grupos con la prueba $U$ de Mann-Whitney, en cuestión de tecnoansiedad, el grupo de secundaria difirió significativamente del de licenciatura $(U=16969.00, p=.001)$ y del de 
posgrado $(U=3584.00, p<.001)$, y el de técnico/bachillerato también difirió del de posgrado $(U=8042.00, p=.007)$; respecto a la tecnofatiga, el grupo de secundaria difirió significativamente del de técnico/bachillerato $(U=4266, p$ $=.043)$, del de licenciatura $(U=13770.00, p<.001)$ y del de posgrado $(U=$ $3225.50, p<.001)$, mientras que el grupo técnico/bachillerato difirió significativamente del de licenciatura $(U=33412, p<.001)$ y del de posgrado $(U=$ 7908.00, p =.004); en la tecnoadicción se observó que el grupo de secundaria difirió significativamente del de licenciatura $(U=16274.50, p<.001)$ y del de posgrado $(U=3879.50, p=.001)$, así como el grupo técnico/bachillerato con respecto al de licenciatura $(U=35833.50, p=.016)$ y al de posgrado $(U=$ $8528.50, p=.049)$. Los grupos de licenciatura y posgrado no difirieron significativamente en ninguna de las variables (véase Figura 1, 2 y 3 ).



Figura 1. Relación entre la escolaridad y la tecnoansiedad

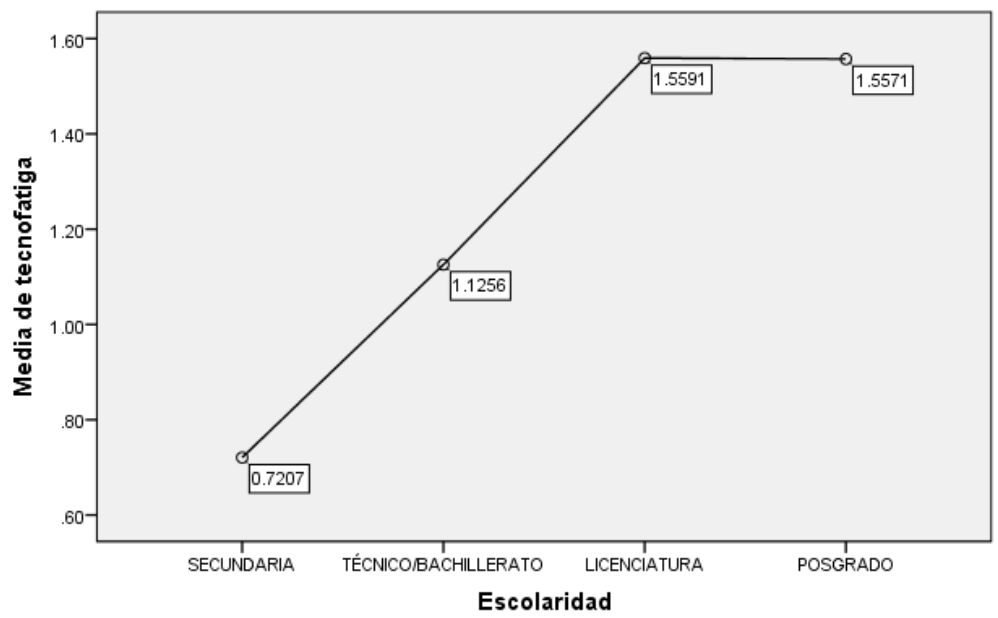

Figura 2. Relación entre la escolaridad y la tecnofatiga 


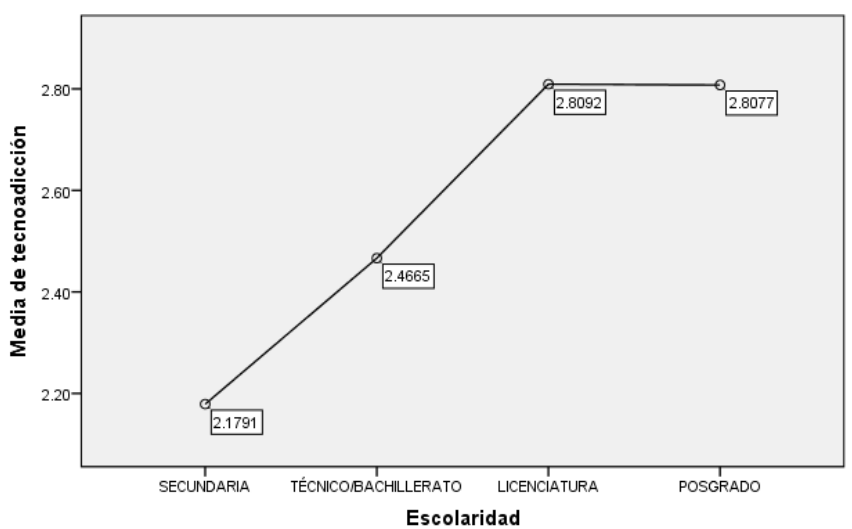

Figura 3. Relación entre la escolaridad y la tecnoadicción

Con base en los puntajes de los factores de tecnoestrés (tecnoansiedad, tecnofatiga y tecnoadicción) comparados por estado civil, se encontró diferencias significativas en el factor tecnoadicción, los solteros obtuvieron puntajes significativamente más altos que los casados. Se encontró una significancia marginal en tecnofatiga, siendo los participantes solteros quienes presentaron mayores puntajes en ese factor (véase Tabla 6).

Tabla 6

Relación entre el estado civil y los factores de tecnoestrés

\begin{tabular}{ccc}
\hline CATEGORÍAS & MEDIAS (DS) & $\begin{array}{c}\text { PRUEBA ESTADÍSTICA } \\
\text { U DE MANN-WHITNEY }\end{array}$ \\
\hline TECNOANSIEDAD & & \\
\hline $\begin{array}{c}\text { Soltero } \\
\text { Casado }\end{array}$ & $0.99(0.89)$ & $U=82348.50, \mathrm{p}=.453$ \\
\hline TECNOFATIGA & $0.93(0.86)$ & \\
\hline $\begin{array}{c}\text { Soltero } \\
\text { Casado }\end{array}$ & $\mathbf{1 . 5 3 ( 1 . 4 2 )}$ & $U=78329.00, \mathrm{p}=.058$ \\
\hline TECNOADICCIÓN & $1.31(1.29)$ & \\
Soltero & & $U=76121.50, \mathrm{p}=. \mathbf{0 1 2}$ \\
Casado & $\mathbf{2 . 8 1 ( 1 . 3 9 )}$ \\
\hline
\end{tabular}

Al comparar los puntajes de los tres factores de tecnoestrés por la ocupación de los participantes (trabajador, ama de casa, estudiante, becario, pasante, prestador de servicio), al aplicar la prueba de Kruskal-Wallis se encontraron diferencias significativas solo para tecnoadicción $\left[\chi^{2(2)}=4.698, p=.008\right]$, siendo las amas de casa quienes obtuvieron mayores puntajes $(U=1.80, S x=$ 1.33) que los trabajadores $(U=1.38, S x=1.36)$ y los estudiantes $(U=1.70, S x$ =1.57). Al hacer comparaciones por pares con la prueba $U$ de Mann Whitney, el grupo de trabajadores difirió significativamente del de estudiantes $(\mathrm{U}=$ 34969.00, $p=.010$ ). 
No se encontraron diferencias significativas por generación (Babyboomers, Generación X, Millennials y Generación Z), con respecto al tecnoestrés. Asimismo, los índices de correlación Rho de Spearman entre la edad y los puntajes de los factores de tecnoestrés resultaron cercanos a cero $y$, por tanto, no significativos (con tecnoansiedad, Rho $=.013$; con tecnofatiga, Rho $=-.026, \mathrm{y}$ con tecnoadicción, Rho $=-.054)$.

\subsection{Relación de variables laborales con tecnoestrés}

De acuerdo con la prueba de Kruskal-Wallis, la variable nivel del puesto desempeñado afectó significativamente el puntaje de tecnoansiedad $\left[\chi^{2(4)}=18.105\right.$, $p=.001]$, de tecnofatiga $\left[\chi^{2(4)}=64.555, p<.001\right]$ y de tecnoadicción $\left[\chi^{2(3)}=\right.$ $43.5222, \mathrm{p}<.001$ ]. Se encontró que los directivos y los empresarios independientes presentaron mayor tecnoansiedad, tecnofatiga y tecnoadicción. Al hacer comparaciones de pares de grupos con la prueba de Mann-Whitney, el grupo del nivel de puesto operativo, el cual obtuvo los puntajes más bajos en los tres factores, difirió significativamente del resto; en tecnoansiedad este grupo difirió del de coordinación $(U=40499.50, p=<$. .001), del de mandos medios $(U=22558.00, p=.025)$ y del de directivos $(U=5430.50, p=.040)$; respecto a la tecnofatiga, el grupo del nivel de puesto operativo difirió significativamente del de coordinación $(U=33948.50, p<.001)$, del de mandos medios $(U=20472.50, p<.001)$, de los directivos $(U=4567.50, p=.001)$ y de los empresarios independientes $(U=6671.50, p<.001)$;

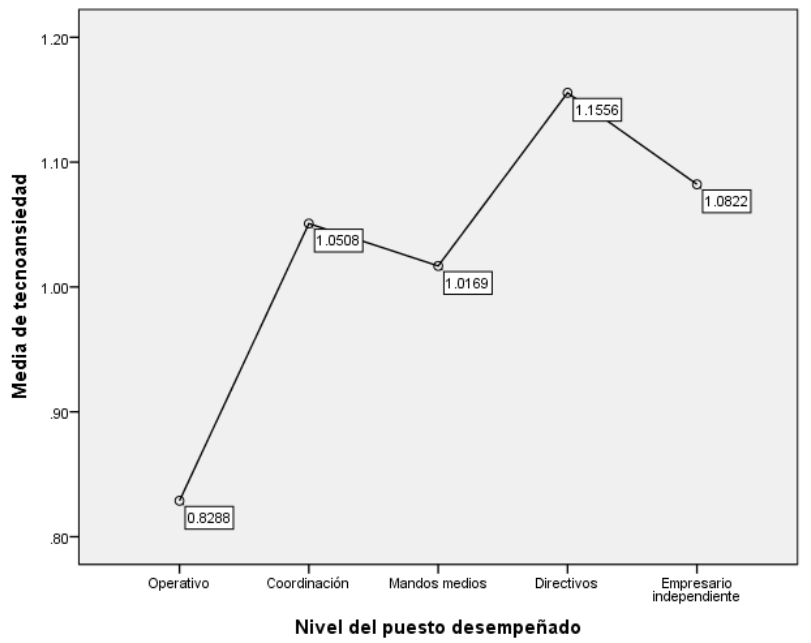

Figura 4. Relación entre la ocupación y la tecnoansiedad

en la tecnoadicción se observó que el grupo del nivel de puesto operativo difirió significativamente del de coordinación $(U=38136.50, p<.001)$, del de mandos medios $(U=19418.50, p<.001)$, de los directivos $(U=4196.00$, 
$p<.001)$ y de los empresarios independientes $(U=8491.50, p=.002)$. Los puestos no operativos no difirieron significativamente entre sí (véase Figuras $4,5$ y 6$)$.

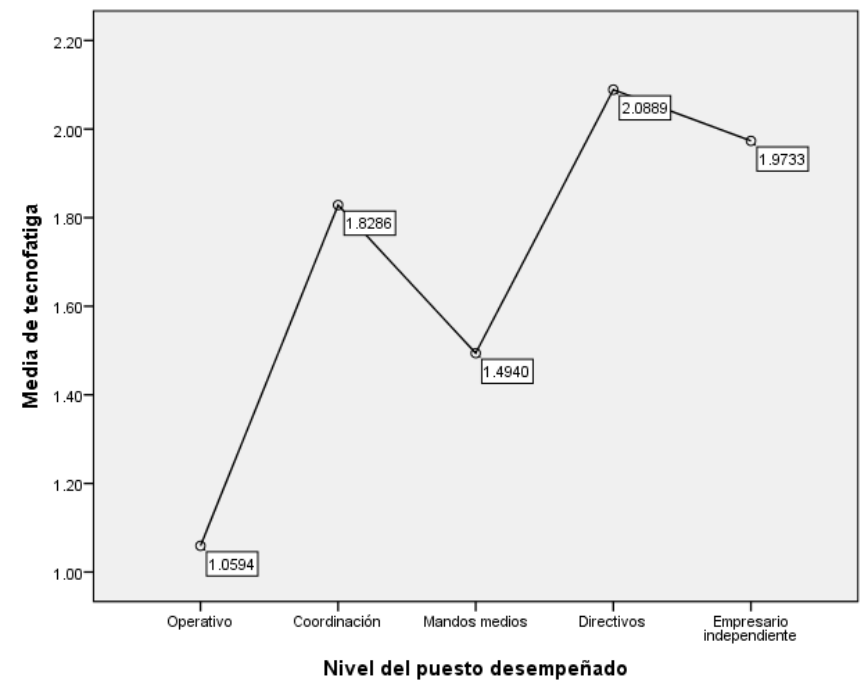

Figura 5. Relación entre la ocupación y la tecnofatiga

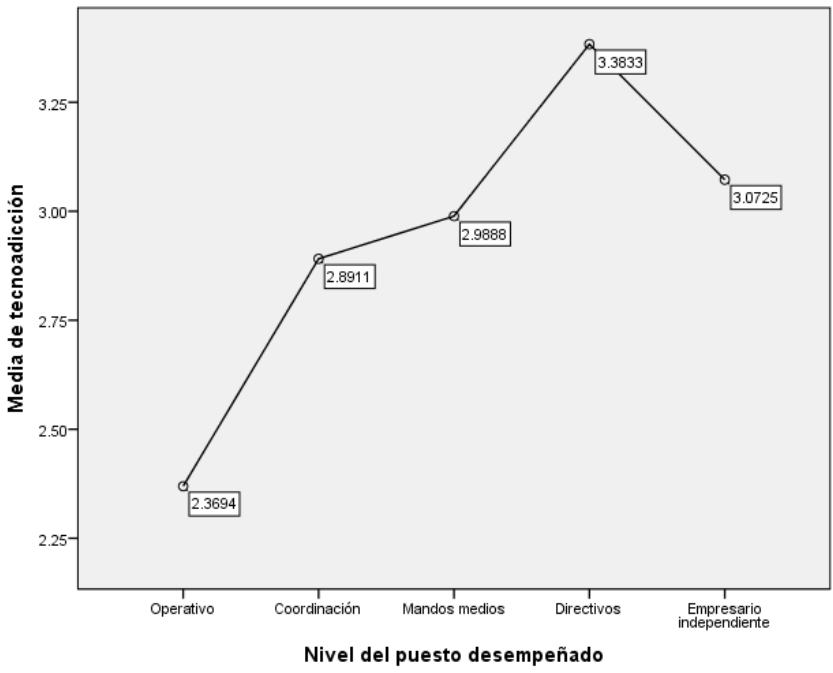

Figura 6. Relación entre la ocupación y la tecnoadicción

Al comparar los puntajes de los factores de tecnoestrés (tecnoansiedad, tecnofatiga y tecnoadicción) por antigüedad en el trabajo (menos de un año, entre 1 y 5 , entre 6 y 10, más de 10 años), se encontraron diferencias significativas únicamente para tecnofatiga, siendo los trabajadores con menos de un año laborando los que obtuvieron mayores puntajes. Al hacer comparaciones por pares de grupos, en cuestión de tecnofatiga el grupo de trabajadores con menos de un año laborando difirió significativamente del grupo 
que tenía entre 6 y 10 años trabajando $(p=.003)$, también el grupo que tenía entre 1 y 5 años de antigüedad difirió significativamente del grupo que tenía entre 6 y 10 en su trabajo $(p=.006)$ (véase Tabla 7).

Tabla 7.

Relación entre la antigüedad en el trabajo y la tecnofatiga

\begin{tabular}{|c|c|c|c|}
\hline CATEGORÍAS & MEDIAS (DS) & $\begin{array}{l}\text { PRUEBA ESTADÍSTICA } \\
\text { KRUSKAL-WALLIS }\end{array}$ & $\begin{array}{c}\text { PRUEBAS } \\
\text { U MANN-WHITNEY }\end{array}$ \\
\hline & & TECNOFATIGA & \\
\hline $\begin{array}{l}\text { Menos de un año } \\
\text { Entre } 1 \text { y } 5 \\
\text { Entre } 6 \text { y } 10 \\
\text { Más de } 10\end{array}$ & $\begin{array}{l}1.49(1.37) \\
1.46(1.38) \\
1.07(1.24) \\
1.28(1.38)\end{array}$ & $\chi^{2}(3)=11.213, p=.011$ &  \\
\hline
\end{tabular}

n.s. = no significativo

Respecto a los puntajes de los factores de tecnoestrés (tecnoansiedad, tecnofatiga y tecnoadicción) comparados por tipo de empresa, se encontraron diferencias significativas por tecnoansiedad, siendo los pertenecientes al sector público quienes obtuvieron puntajes más altos, en contraste con aquellos del sector privado (véase Tabla 8).

Tabla 8.

Relación entre el tipo de empresa y los factores de tecnoestrés

\begin{tabular}{lll}
\hline CATEGORÍAS & MEDIAS (DS) & PRUEBA ESTADÍSTICA \\
\hline & TECNOANSIEDAD & \\
\hline Sector público & $1.05(0.97)$ & $\mathrm{U}=61348.00, \mathrm{p}=. \mathbf{0 4 9}$ \\
Sector privado & $0.89(0.84)$ & \\
\hline & TECNOFATIGA & $\mathrm{U}=64757.00, \mathrm{p}=.376$ \\
\hline Sector público & $1.47(1.41)$ & \\
\hline Sector privado & $1.36(1.35)$ & \\
\hline & & \\
\hline & TECNOADICCIÓN & \\
\hline Sector público & $2.63(1.33)$ & \\
\hline Sector privado & $2.65(1.41)$ & \\
\hline
\end{tabular}

\subsection{Relación de la frecuencia de uso de TIC con tecnoestrés}

La frecuencia de uso de TIC no afectó significativamente el puntaje de tecnoansiedad, tecnofatiga ni tecnoadicción (véase Tabla 9). 
Tabla 9.

Relación entre la frecuencia de uso de las TIC y los factores de tecnoestrés

\begin{tabular}{|c|c|c|}
\hline CATEGORÍAS & MEDIAS (DS) & $\begin{array}{l}\text { PRUEBA ESTADÍSTICA } \\
\text { KRUSKAL-WALLIS }\end{array}$ \\
\hline \multicolumn{3}{|c|}{ TECNOANSIEDAD } \\
\hline $\begin{array}{c}\text { Nunca } \\
\text { Un par de veces al mes } \\
\text { Una vez a la semana } \\
\text { Un par de veces a la semana } \\
\text { Todos los días }\end{array}$ & $\begin{array}{l}0.62(0.53) \\
1.52(1.44) \\
0.96(0.80) \\
0.93(0.96) \\
0.94(0.87)\end{array}$ & $\chi^{2}(3)=1.595, p=.660$ \\
\hline \multicolumn{3}{|c|}{ TECNOFATIGA } \\
\hline $\begin{array}{c}\text { Nunca } \\
\text { Un par de veces al mes } \\
\text { Una vez a la semana } \\
\text { Un par de veces a la semana } \\
\text { Todos los días }\end{array}$ & $\begin{array}{l}1.15(0.85) \\
1.72(1.74) \\
1.33(0.99) \\
1.35(1.38) \\
1.43(1.40)\end{array}$ & $\chi^{2}(3)=0.607, p=.895$ \\
\hline \multicolumn{3}{|c|}{ TECNOADICCIÓN } \\
\hline $\begin{array}{c}\text { Nunca } \\
\text { Un par de veces al mes } \\
\text { Una vez a la semana } \\
\text { Un par de veces a la semana } \\
\text { Todos los días }\end{array}$ & $\begin{array}{l}2.59(1.26) \\
2.65(1.21) \\
2.77(1.21) \\
2.66(1.36) \\
2.71(1.43)\end{array}$ & $\chi^{2}(3)=0.074, p=.995$ \\
\hline
\end{tabular}

\section{DISCUSIÓN}

Hoy en día nos enfrentamos a la tecnología en cualquier lugar y en todo momento. La pregunta está en cómo las personas han reaccionado ante este fenómeno que comenzó tan solo hace algunos años. La investigación científica en otros países ha llevado a identificar tres experiencias diferentes durante la interacción con la tecnología, estas son la ansiedad, la fatiga y la adicción a las TIC, conformando lo que se conoce como tecnoestrés.

Desde la literatura se identificó que las consecuencias de sufrir tecnoestrés impactan tanto a nivel personal (fisiológico-psicosocial), a nivel profesional (como estudiante), y a nivel laboral (como trabajador en las organizaciones), surgiendo así un tema relevante a estudiar.

La presente investigación puso, particularmente, bajo estudio la relación entre variables sociodemográficas, laborales y la frecuencia del uso de las TIC, con el tecnoestrés. Los resultados que se obtuvieron permiten aproximarse a este fenómeno en sus tres experiencias (tecnoansiedad, tecnofatiga y tecnoadicción), entendiendo que cada una tiene sus propias características, pero que una persona puede experimentar más de una a la vez. 
En primer lugar, los datos del presente análisis coinciden con aquellos estudios que afirman que son las mujeres las que manifiestan mayores niveles de fatiga al usar las TIC (Salanova et al., 2007; Baloglu \& Cevik, 2008; Carlotto et al., 2017; Picón et al., 2017) siendo que, tentativamente ellas muestran actitudes más negativas y sentimientos de poca capacidad para emplearlas. A pesar de que se ha reportado que los hombres son más compulsivos con el uso de las tecnologías (Lee et al., 2014), los resultados en esta ocasión no mostraron diferencias significativas por tecnoadicción entre hombres y mujeres.

Los puntajes arrojaron que no hay diferencias significativas en cuanto a la edad, resultado que es consistente con Khasawneh (2018), quien afirma que tanto jóvenes como adultos han aceptado el uso cotidiano y frecuente de la tecnología. Con respecto a la variable generación, Coppari et al. (2017) argumentan que los millennials y centennials tienen un desarrollo más cercano con la tecnología, son quienes la utilizan en mayor medida y están mejor adaptados a los cambios que esta representa en comparación con la generación $X$. Sin embargo, lo que se encontró en este estudio es que no hay diferencias significativas entre las generaciones babyboomers $X, Y$ y $Z$. Se sugiere someter a estudio la comparación entre estas cuatro generaciones, aumentando el número de participantes BabyBoomer, ya que las personas de esta generación podrían exhibir diferencias significativas debido a la poca cercanía que tuvieron con la tecnología en el pasado.

Un estudio realizado en Brasil encontró que los trabajadores casados, en comparación con los solteros, padecen más fatiga a causa del uso de la tecnología (Carlotto et al., 2017). Esta información contradice lo arrojado por esta investigación, debido a que los solteros obtuvieron una diferencia marginal en lo que respecta a la tecnofatiga. Además, se encontró que los solteros muestran niveles más altos de tecnoadicción que los casados, esto podría deberse al mayor uso que hacen de las redes sociales.

Se encontró que a mayor escolaridad, mayor tecnoansiedad, tecnofatiga y tecnoadicción, información contradictoria a lo reportado por Ragu-Nathan et al. (2008), cuyo estudio afirma que el tecnoestrés disminuye conforme el nivel educativo aumenta. Este resultado puede derivarse de que un mayor nivel de escolaridad implica un aumento de tareas y de su complejidad, las cuales para su realización demandan el uso de las tecnologías.

También, se obtuvieron diferencias significativas en tecnoadicción según la ocupación, siendo las amas de casa quienes obtuvieron puntajes más altos que los trabajadores y los estudiantes, y una diferencia significativa entre estos 
dos últimos grupos, resultados consistentes con la investigación de Sonya (2003), quien concluye que las encargadas de las labores del hogar presentan mayores niveles de tecnoestrés, en comparación con los estudiantes.

El análisis de los datos de esta investigación reveló que los directivos y los empresarios independientes presentaron mayor tecnoansiedad, tecnofatiga y tecnoadicción en comparación con los operativos, coordinadores y mandos medios. Este dato podría ser el resultado de que a mayor nivel de puesto, se tiene mayor interacción con la tecnología y los procedimientos que se deben hacer con esta son más complejos.

El estudio de Ragu-Nathan et al. (2008), reconoció que la antigüedad laboral puede fungir como un factor protector del trabajador ante situaciones estresantes. Este aspecto coincide con los resultados arrojados ya que se encontraron diferencias significativas para tecnofatiga, siendo los trabajadores con menos de un año de antigüedad los que obtuvieron mayores puntajes.

Por otra parte, no se encontró estudio alguno que reportara la relación entre el tipo de empresa (pública o privada) y el tecnoestrés en los trabajadores; es por esto que el presente estudio se interesó en realizar este análisis, del cual se encontraron diferencias significativas únicamente para tecnoansiedad, siendo el grupo perteneciente al sector público el que obtuvo puntajes más altos.

Finalmente, se realizó el análisis de los datos para identificar la relación de la frecuencia del uso de las TIC y el tecnoestrés. No se obtuvieron diferencias significativas. Este resultado se añade a aquellos estudios que, por un lado afirman que entre más uso de las tecnologías, se tiene mayor experiencia en su manejo y menor ansiedad (Çoklar \& Sahin, 2011; Picón et al., 2017), y que, por otro lado, afirman que cuando en gran parte de la jornada de trabajo (más del $75 \%$ del tiempo) se usan las TIC, se presenta tecnoestrés (Brooks, 2015; Carlotto et al., 2017).

El conocer si ciertas características sociodemográficas, laborales, e incluso la frecuencia con la que se usan las TIC, impactan en los niveles de tecnoestrés es importante porque permite que las personas identifiquen los grupos a los que pertenecen y conozcan cómo es que estos se relacionan con el tecnoestrés, de tal forma que esta información les ayude a mitigar la ansiedad, fatiga o adicción que el uso de las tecnologías propicia. Asimismo, se busca que las organizaciones (educativas, laborales, etc.) cuenten con información 
suficiente al momento de tomar decisiones, como lo es el incorporar nuevas tecnologías a las actividades diarias de las personas.

El análisis psicométrico del instrumento arrojó un alpha de Cronbach de 0.86 para la escala total y para los factores fue superior a 0.80 , lo que implica que ya se cuenta con dos escalas que servirán como herramientas para el estudio del fenómeno del tecnoestrés y de la tecnoadicción en México.

Cabe señalar, que aún no existe suficiente evidencia teórica con respecto al tecnoestrés, ya que los resultados siguen siendo poco consistentes entre unos y otros. Particularmente, en México, no se cuentan con investigaciones sobre el tema. Se sugiere realizar más estudios, tanto de tipo cuantitativo como cualitativo, que ayuden a tener una perspectiva más cercana de la experiencia de los usuarios de la tecnología, y de quienes padecen tecnoestrés.

Esta investigación tiene gran importancia teórica debido a que ha encontrado resultados que representan las primeras aportaciones sobre el estudio del tecnoestrés en la cultura mexicana, los cuales buscan entender este fenómeno y abordar las variables que se encuentran relacionadas con las distintas experiencias del mismo.

El estudio está sujeto a limitaciones, se debe considerar que lo que se buscaba era obtener una muestra diversa lo que provocó que los grupos a comparar, según las distintas variables, no contaran con el mismo número de personas. Como desafío futuro próximo, se plantea seguir analizando el rol que juegan estas y otras variables en los niveles de tecnoestrés, teniendo nuevos criterios de inclusión que garanticen el mismo número de personas por grupo a comparar. Asimismo, el medio a través del cual se aplicó el instrumento fue el formulario de Google, futuras investigaciones podrían implementar uno distinto.

Finalmente, se invita a crear y proponer programas de intervención, que permitan reducir el uso desadaptativo al estrés causado por las tecnologías.

Conflictos de interés: No se presenta ningún conflicto de interés en la investigación que pudiera comprometer los resultados obtenidos; no hubo fuente de financiamiento para llevarla a cabo y no existió vínculo alguno entre las investigadoras y los participantes.

Agradecimientos: A la Facultad de Psicología de la Universidad Nacional Autónoma de México, por las facilidades otorgadas para la realización del presente estudio.

Financiamiento: La presente investigación no contó con ningún tipo de financiamiento. 


\section{REFERENCIAS}

Abrahams, D. A. (2010). Technology adoption in higher education: a framework for identifying and prioritising issues and barriers to adoption of instructional technology. Journal of Applied Research in Higher Education, 2(2), 34-49. https:// doi.org/10.1108/17581184201000012

Agogo, D., \& Hess, T. J. (2015). Technostress and technology induced state anxiety: Scale development and implications. In Thirty Sixth International Conference on Information Systems, pp.1-11. Fort Worth, Texas, EUA. https://agogodavid.com/ wp-content/uploads/2015/06/Agogo-and-Hess-ICIS-2015-Submit.pdf

Ahmad, S., Rafiq, M., \& Ahmad, S. (2018). Gender disparities in the use of internet among graduate students of a developing society: A case of Pakistani universities. Global Knowledge, Memory and Communication, 67(4/5), 226-243. https://doi. org/10.1108/GKMC-11-2017-0092

Alfaro de Prado, A. (2008). Nuevas tecnologías y nuevos riesgos laborales: Estrés y tecnoestrés. Revista digital de salud y seguridad en el trabajo, (1), 123-155. http://rabida.uhu.es/dspace/bitstream/handle/10272/3414/b15756531.pdf?sequence $=1$

Allan, J., \& Lawless, N. (2003). Stress caused by on-line collaboration in e-learning: a developing model. Education + Training, 45(8/9), 564-572. https://doi. org/10.1108/00400910310508955

Al-Yafi, K., El-Masri, M., \& Tsai, R. (2018). The effects of using social network sites on academic performance: the case of Qatar. Journal of Enterprise Information Management, 31(3), 446-462. https://doi.org/10.1108/JEIM-08-2017-0118

Baloglu, M., \& Cevik, V. (2008). Multivariate effects of gender, ownership, and the frequency of use on computer anxiety among high school students. Computers in Human Behavior, 24, 2639-2648. https://doi.org/10.1016/j.chb.2008.03.003

Brooks, S. (2015). Does personal social media usage affect efficiency and well-being? Computers in Human Behavior, 46, 2-37. https://doi.org/10.1016/j. chb.2014.12.053

Brooks, S., \& Califf, C. (2017). Social media induced technostress: Its impact on the job performance of it professionals and the moderating role of job characteristics. Computer Networks 114, 143-153. https://doi.org/10.1016/j. comnet.2016.08.020

Cao, X., Masood, A., Luqman, A., \& Ali, A. (2018). Excessive use of mobile social networking sites and poor academic performance: Antecedents and consequences from stressor-strain out come perspective. Computers in Human Behavior, 85, 163-174. https://doi.org/10.1016/j.chb.2018.03.023

Cao, X., \& Sun, J. (2018). Exploring the effect of overload on the discontinuous intention of social media users: An S-O-R perspective. Computers in Human Behavior, 81, 10-18. https://doi.org/10.1016/j.chb.2017.11.035

Cao, X., \& Yu, L. (2019). Exploring the influence of excessive social media use at work: A three dimension usage perspective. International Journal of Information Management, 46, 83-92. https://doi.org/10.1016/j.ijinfomgt.2018.11.019

Carlotto, M., Welter, W. G., \& Jones, A. (2017). Technostress, Career Commitment, Satisfaction with Life, and Work-Family Interaction among Workers in Information and Communication Technologies. Actualidades en Psicología, 31(122), 91-102. http://dx.doi.org/10.15517/ap.v31i122.22729

Cazares, V. M., \& Villavicencio, E. (2019). Adaptación de dos escalas para medir tecnoestrés y tecnoadicción en una población laboral mexicana (tesis de licenciatura). Facultad de Psicología, UNAM, Ciudad de México. http://132.248.9.195/ ptd2019/mayo/0788864/Index.html 
Çoklar, A., \& Sahin, Y. (2011). Technostress levels of social network users based on ICTs in Turkey. European Journal of Social Sciences, 23(2), 171-182. https:// www.researchgate.net/publication/287599284_Technostress_levels_of_social_ network_users_based_on_ICTS_in_Turkey

Coppari, N., Bagnoli, L., Codas, G., López, H., Martínez, U., Martínez, L., \& Montanía, M. (2018). Validez y confiabilidad del cuestionario de tecnoestrés en estudiantes paraguayos. Perspectivas en Psicología, 15(2), 40-55. http://www.seadpsi.com. ar/revistas/index.php/pep/article/view/412

Coppari, N., Bagnoli, L., Codas, G., Montanía, M., Martínez, Ú., \& López Humada, H. (2017). Uso de Tecnologías de la Comunicación e Información y Tecnoestrés en Estudiantes Paraguayos: su relación con la edad. Cuadernos de Neuropsicología/ Panamerican Journal of Neuropsychology, 11(3), 166-181. http://www.cnps.cl/ index.php/cnps/article/view/306/325

De Wet, W., Koekemoer, E., \& Nel, J. A. (2016). Exploring the impact of information and communication technology on employees' work and personal lives. SA Journal of Industrial Psychology, 42(1), 1330. http://dx.doi.org/10.4102/sajip. v42i1.1330

Dhanapal, S., Vashu, D., \& Subramaniam, T. (2015). Perceptions on the challenges of online purchasing: a study from "baby boomers", generation " $X$ " and generation " $Y$ " point of views. Contaduría y Administración, 60(1), 107-132. https://doi. org/10.1016/j.cya.2015.08.003

Dhir, A., Yossatorn, Y., Kaur, P., \& Chen, S. (2018). Online social media fatigue and psychological wellbeing -A study of compulsive use, fear of missing out, fatigue, anxiety and depression. International Journal of Information Management, 40, 141-152. https://doi.org/10.1016/j.ijinfomgt.2018.01.012

Duke, É., \& Montag, C. (2017). Smartphone addiction, daily interruptions and self-reported productivity. Addictive Behaviors Reports, 6, 90-95. https://doi. org/10.1016/j.abrep.2017.07.002

Gaspar, H. S. (2016). Bases psicosociales del uso del smartphone en jóvenes: un análisis motivacional y cross-cultural (Tesis Doctoral). Facultad de ciencias de la información, Universidad Complutense de Madrid. http://eprints.ucm. es/35447/1/T36788.pdf

Gaudioso, F., Turel, O., \& Galimberti, C. (2017). The mediating roles of strain facets and coping strategies in translating techno-stressors into adverse job outcomes. Computers in Human Behavior, 69, 189-196. https://doi.org/10.1016/j. chb.2016.12.041

Grant, C. A., Wallace, L. M., Spurgeon, P. C., Tramontano, C., \& Charalampous, M. (2019). Construction and initial validation of the E-Work Life Scale to measure remote e-working. Employee Relations, 41(1), 16-33. https://doi.org/10.1108/ ER-09-2017-0229

Gobierno de la Ciudad de México. (Febrero, 2018). Activan internet gratuito en línea 1 del metro. https://www.metro.cdmx.gob.mx/comunicacion/nota/activan-internet-gratuito-en-linea-1-del-metro

Gobierno de la Ciudad de México. (Febrero, 2019). México Conectado. https:// mexicoconectado.gob. $\mathrm{mx}$ /

Guillén, Z. F. (2016). Relación entre los cinco grandes rasgos de personalidad y las dimensiones del tecnoestrés. https://repositorio.comillas.edu/xm/ui/bitstream/ handle/11531/9718/TFM000406. pdf?sequence=1\&isAllowed=y

Haddara, M., \& Hetlevik, T. (2016). Investigating the Effectiveness of Traditional Support Structures \& Self-Organizing Entities within the ERP Shakedown 
Phase. Procedia Computer Science, 100, 507-516. https://doi.org/10.1016/j. procs.2016.09.189

Hernández, R., Fernández, C., \& Baptista, M. (2014). Metodología de la investigación. (6a ed.). México: Mc. Graw Hill. https://www.academia.edu/28050831/Metodologia_de_la_Investigacion___Sampieri_6ta_edicion_

Hill, R., Davies, P. B., \& Williams, M. D. (2008). Older people and internet engagement: Acknowledging social moderators of internet adoption, access and use. Information Technology \& People, 21(3), 244-266. https://doi. org/10.1108/09593840810896019

Hsiao, K-L. (2017). Compulsive mobile application usage and technostress: the role of personality traits. Online Information Review, 41(2), 272-295. https://doi. org/10.1108/OIR-03-2016-0091

Hsiao, K-L., Shu, Y., \& Huang, T-C. (2017). Exploring the effect of compulsive social app usage on technostress and academic performance: Perspectives from personality traits. Telematics and Informatics, 34, 679-690. https://doi.org/10.1016/j. tele.2016.11.001

Hughes, N., \& Burke, J. (2018). Sleeping with the frenemy: How restricting 'bedroom use' of smartphones impacts happiness and wellbeing. Computers in Human Behavior, 85, 236-244. https://doi.org/10.1016/j.chb.2018.03.047

Jung, Y., Pawlowski, S. D., \& Kim, H-W. (2017). Exploring associations between young adults' facebook use and psychological well-being: A goal hierarchy approach. International Journal of Information Management, 37, 1391-1404. https://doi. org/10.1016/j.ijinfomgt.2016.10.005

Kerlinger, F. N., \& Lee, H. B. (2002). Investigación del comportamiento: métodos de investigación en ciencias sociales. (4a ed.). México: McGraw-Hill / Interamericana Editores. https://www.academia.edu/6753714/Investigacion_Del_Comportamiento___Kerlinger_Fred_N_PDF

Khasawneh, O. Y. (2018a). Technophobia without boarders: The influence of technophobia and emotional intelligence on technology acceptance and the moderating influence of organizational climate. Computers in Human Behavior, 88, 210-218. https://doi.org/10.1016/j.chb.2018.07.007

Khasawneh, O. Y. (2018b). Technophobia: Examining its hidden factors and defining it. Technology in Society, 54, 93-100. https://doi.org/10.1016/j.techsoc.2018.03.008

Khatri, V., Samuel, B. M., \& Dennis, A. R. (2018). System 1 and System 2 cognition in the decision to adopt and use a new Technology. Information \& Management, 55, 709-724. https://doi.org/10.1016/j.im.2018.03.002

Khuntia, J., Tanniru, M., \& Weiner, J. (2015). Juggling digitization and technostress: The case of alert fatigues in the patient care system implementation. Health Policy and Technology, 4, 364-377. https://doi.org/10.1016/j.hlpt.2015.08.005

Krishnan, S. (2017). Personality and espoused cultural differences in technostress creators. Computers in Human Behavior, 66, 154-167. https://doi.org/10.1016/j. chb.2016.09.039

Lee, Y. K., Chang, C. T., Lin, Y., \& Cheng, Z. H. (2014). The dark side of smartphone usage: Psychological traits, compulsive behavior and technostress. Computers in human behavior, 31, 373-383. https://doi.org/10.1016/j.chb.2013.10.047

Llorens, S., Salanova, M., \& Ventura, M. (2011). Guías de intervención. Tecnoestrés. Madrid: Editorial Síntesis. http://www.want.uji.es/wp-content/ uploads/2017/11/2011_Llorens-Salanova-Ventura-Tecnoestres.pdf

Loderer, K., Pekrun, R., \& Lester, J. C. (2018). Beyond cold technology: A systematic review and meta-analysis on emotions in technology-based learning 
environments. Learning and Instruction, 1-15. https://doi.org/10.1016/j. learninstruc.2018.08.002

Loiacono, E, \& McCoy, S. (2018). When did fun become so much work: The impact of social media invasiveness on continued social media use. Information Technology \& People, 31(4), 966-983. https://doi.org/10.1108/ITP-10-2016-0239

Luqman, A., Cao, X., Ali, A., Masood, A., \& Yu, L. (2017). Empirical investigation of Facebook discontinues usage intentions based on SOR paradigm. Computers in Human Behavior, 70, 544-555. https://doi.org/10.1016/j.chb.2017.01.020

Martínez-Corcoles, M., Teichmann, M., \& Murdvee, M. (2017). Assessing technophobia and technophilia: Development and validation of a questionnaire. Technology in Society, 51, 183-188. https://doi.org/10.1016/j.techsoc.2017.09.007

Mattila, M., Karjaluoto, H., \& Pento, T. (2003). Internet banking adoption among mature customers: early majority or laggards? Journal of Services Marketing, 17(5), 514-528. https://doi.org/10.1016/j.techsoc.2017.09.007

McEwen, B. S. (2006). Protective and damaging effects of stress mediators: central role of the brain. Dialogues in Clinical Neuroscience, 8(4), 367-381. https://www. researchgate.net/publication/6513840_Protective_and_Damaging_Effects_of_ Stress_Mediators_Central_Role_of_the_Brain

México Digital. (2014). Programa de Inclusión y Alfabetización Digital (PIAD). https:// www.gob.mx/mexicodigital/articulos/programa-de-inclusion-y-alfabetizacion-digital-piad

Montag, C., Błaszkiewicz, K., Sariyska, R., Lachmann, B., Andone, I., Trendafilov, B., \& Markowetz, A. (2015). Smartphone usage in the 21st century: Who is active on WhatsApp? BMC Research Notes, 8(1), 331. https://doi.org/10.1186/s13104015-1280-z

Mueller, M. (2006). Keep Breathing: Coping with Technology. Library Hi Tech News, 23(5), 27-30. https://doi.org/10.1108/07419050610689059

Oh, S. T., \& Sungbum, P. (2016). A study of the connected smart worker's TechnoStress. Procedia Computer Science, 91, 725-733. https://doi.org/10.1016/j. procs.2016.07.065

Picón, C., Toledo, S., \& Navarro, V. (2017). Tecnoestrés: Identificación y prevalencia en el personal docente de la Facultad de Medicina de la Universidad Nacional del Nordeste. Revista de la Facultad de Medicina, 36(3), 41-51. http://revistas.unne. edu.ar/index.php/rem/article/view/2309

Quinn, B. (2001). The medicalisation of online behavior. Online Information Review, 25(3), 173-180. https://doi.org/10.1108/14684520110395308

Ragu-Nathan, T. S., Tarafdar, M., Ragu-Nathan, B. S., \& Tu, Q. (2008). The consequences of technostress for end users in organizations: Conceptual development and empirical validation. Information Systems Research, 19, 417-433. https://doi. org/10.1287/isre.1070.0165

Riedl, R. (2013). On the biology of technostress: literature review and research agenda. The DATA BASE for Advances in Information Systems, 44, 18-55. https:// doi.org/10.1145/2436239.2436242

Salanova, M., Cifre, E., \& Martin, P. (2004). Information technology implementation styles and their relation with workers' subjective well-being. International Journal of Operations \& Production Management, 24(1), 42-54. https://doi. org/10.1108/01443570410510988

Salanova, M., \& Llorens, S. (2009). Exposure to Information and Communication Technology and its relationship to work engagement. Ciencia y Trabajo, 32, 55-63. https://pdfs.semanticscholar.org/f057/9b1a7a7cf6cb1f1165f7b6e02d50ef7e761b.pdf 
Salanova, M., Llorens, S., Cifre, E., \& Nogareda, C. (2007). Tecnoestrés: concepto, medida e intervención psicosocial. Nota técnica de prevención, 730. https://www. insst.es/documents/94886/196283/NTP+730+Tecnoestr\%C3\%A9s.+concepto\%2C+medida+e+intervenci\%C3\%B3n+psicosocial.pdf/a180de9a-bb95-4a10-a403392f1b96a20b?version=1.0

Salanova, M., \& Schaufeli, W. (2000). Exposure to information technology and its relation to burnout. Behavioral and Information Technology, 19, 385-392. https:// doi.org/10.1080/014492900750000081

Sami, L. K., \& Pangannaiah, N. B. (2006). "Technostress" A literature survey on the effect of information technology on library users. Library Review, 55(7), 429-439. https://doi.org/10.1108/00242530610682146

Seong, T. O., \& Park, S. (2016). A Study of the Connected Smart Worker's TechnoStress. Procedia Computer Science, 91, 725-733. https://doi.org/10.1016/j. procs.2016.07.065

Shao, Z. (2018). Examining the impact mechanism of social psychological motivations on individuals' continuance intention of MOOCs: The moderating effect of gender. Internet Research, 28(1), 232-250. https://doi.org/10.1108/IntR-11-2016-0335

Sigala, M. (2018). New technologies in tourism: From multi-disciplinary to anti disciplinary advances and trajectories. Tourism Management Perspectives, 25, 151-155. https://doi.org/10.1016/j.tmp.2017.12.003

Sonya, G. S. (2003). The Relationship between Computer Skills and the Level of Techno stress among Faculty and Academic Librarians from Selected Institutions within the University System of Georgia [Unpublished Doctoral Thesis]. USA: Georgia Southern University. https://pdfs.semanticscholar.org/61a5/eb0ecccfd60e50d6c944fd44d39462867964.pdf?_ga=2.243505538.609119061.1573452374724809602.1549012725

Steelman, Z. R., \& Soror, A. A. (2017). Why do you keep doing that? The biasing effects of mental states on IT continued usage intentions. Computers in Human Behavior, 73, 209-223. https://doi.org/10.1016/j.chb.2017.03.027

Stich, J., Tarafdar, M., Stacey, P., \& Cooper, C. (2018). E-mail load, workload stress and desired e-mail load: a cybernetic approach. Information Technology \& People, 32(2), 430-452. https://doi.org/10.1108/ITP-10-2017-0321

Tams, S., Legoux, R., \& Leger, P. M. (2018). Smartphone withdrawal creates stress: A moderated mediation model of nomophobia, social threat, and phone withdrawal context. Computers in Human Behavior, 81, 1-9. https://doi.org/10.1016/j. chb.2017.11.026

Teo, T. (2001). Demographic and motivation variables associated with Internet usage activities. Internet Research, 11(2), 125-137. https://doi. org/10.1108/10662240110695089

Thomee, S., Eklof, M., Gustafsson, E., Nilsson, R., \& Hagber, G. M. (2007). Prevalence of perceived stress, symptoms of depression and sleep disturbances in relation to information and communication technology (ICT) use among young adults An explorative prospective study. Computers in Human Behavior, 23, 1300-1321. https://doi.org/10.1016/j.chb.2004.12.007

West, P. T., \& Saker, J. (2012). Computer assisted sales processes in automotive retailing. International Journal of Retail \& Distribution Management, 40(7), 493-509. https://doi.org/10.1108/09590551211239828

Yao, J., \& Cao, X. (2017). The balancing mechanism of social networking overuse and rational usage. Computers in Human, 75, 415-422. https://doi.org/10.1016/j. chb.2017.04.055 
Yan, Z., Guo, X., Lee, M. K. O., \& Vogel, D. R. (2013). A conceptual model of technology features and technostress in telemedicine communication. Information Technology \& People, 26(3), 283-297. https://doi.org/10.1108/ITP-04-2013-0071

Yin, P., Ou, C., Davison, R. M., \& Wu, J. (2018). Coping with mobile technology overload in the workplace. Internet Research, 28(5), 1189-1212. https://doi.org/10.1108/ IntR-01-2017-0016

Yu, B., Ndumu, A., Mon, L. M. \& Fan, Z. (2018). E-inclusion or digital divide: an integrated model of digital inequality. Journal of Documentation, 74(3), 552-574. https://doi.org/10.1108/JD-10-2017-0148

Yu, L., Cao, X., Liu, Z., \& Wang, J. (2018). Excessive social media use at work: Exploring the effects of social media overload on job performance. Information Technology \& People, 31(6), 1091-1112. https://doi.org/10.1108/ITP-10-2016-0237

Zhang, S., Zhao, L., Lu, Y., \& Yang, J. (2016). Do you get tired of socializing? An empirical explanation of discontinuous usage behavior in social network services. Information \& Management, 53, 904-914. https://doi.org/10.1016/j.im.2016.03.006

Esta obra está bajo: Creative commons attribution 4.0 international license. El beneficiario de la licencia tiene el derecho de copiar, distribuir, exhibir y representar la obra y hacer obras derivadas siempre y cuando reconozca y cite la obra de la forma especificada por el autor o el licenciante.

\section{(cc) $\mathbf{B Y}$}

